\title{
Axion Dark Matter Coupling to Resonant Photons via Magnetic Field
}

\author{
Ben T. McAllister, Stephen R. Parker, and Michael E. Tobar ${ }^{\dagger}$ \\ ARC Centre of Excellence for Engineered Quantum Systems, School of Physics, The University of Western Australia, \\ 35 Stirling Highway, Crawley 6009, Western Australia, Australia
}

(Received 17 December 2015; published 21 April 2016)

\begin{abstract}
We show that the magnetic component of the photon field produced by dark matter axions via the two-photon coupling mechanism in a Sikivie haloscope is an important parameter passed over in previous analysis and experiments. The interaction of the produced photons will be resonantly enhanced as long as they couple to the electric or magnetic mode structure of the haloscope cavity. For typical haloscope experiments the electric and magnetic couplings are equal, and this has implicitly been assumed in past sensitivity calculations. However, for future planned searches such as those at high frequency, which synchronize multiple cavities, the sensitivity will be altered due to different magnetic and electric couplings. We define the complete electromagnetic form factor and discuss its implications for current and future dark matter axion searches over a wide range of masses.
\end{abstract}

DOI: 10.1103/PhysRevLett.116.161804

Axions are a type of weakly interacting slim particle originating from the Peccei-Quinn solution to the strong $C P$ problem in QCD [1]. They can be formulated as highly motivated and compelling components of cold dark matter [2-5]. Cosmological constraints provide upper and lower limits on the mass of the axion [6], yet still leave a large area of parameter space to be searched. One of the most mature and sensitive experiments is the Sikivie haloscope [7,8], which exploits the inverse Primakoff effect whereby a magnetic field provides a source of virtual photons in order to induce axion-to-photon conversion via a two photon coupling, with the generated real photon frequency being dictated by the axion mass. This signal is then resonantly enhanced by a cavity structure and resolved above the thermal noise of the measurement system. It has been well established that in a haloscope with an axial dc magnetic field the expected power due to axion-to-photon conversion is given by [7-9]

$$
P_{a}=\left(\frac{g_{\gamma} \alpha}{\pi f_{a}}\right)^{2} \frac{\rho_{a}}{m_{a}} V B_{0}^{2} Q C,
$$

where $g_{\gamma}$ is a dimensionless model-dependent parameter of $\mathcal{O}(1)$ [10-12], $\alpha$ is the fine structure constant, $f_{a}$ is the Peccei-Quinn energy breaking scale, which dictates the axion mass and coupling strength, $m_{a}$ is the axion mass, $\rho_{a}$ is the local density of axions, $V$ is the cavity volume, $B_{0}$ is the applied magnetic field, $Q$ is the cavity quality factor (assuming the bandwidth is greater than the expected spread of the axion signal), and $C$ is the haloscope form factor describing the overlap between the electric field created by the converted axions and the electric field structure of the resonant mode in the cavity.

To date, haloscope searches have excluded some areas of the parameter space $[9,13]$, with further experiments currently under way and future efforts in various stages of planning [14-16]. All of this work fundamentally relies on Eq. (1) to set constraints on $f_{a}$ and hence the mass of the axion and the strength of the axion-photon coupling. In deriving Eq. (1) the coupling of an axion to a photon electric field is explicitly considered in the form factor $C$ and it is then assumed that the corresponding magnetic field coupling is the same. Recent work describes the design of a magnetometer detection experiment enhanced by an $L C$ circuit that utilizes the magnetic field coupling [17]. In this work we consider the coupling of the photon magnetic field directly to a haloscope cavity, and from this we are able to define the complete electromagnetic form factor for the axion haloscope. Revising Eq. (1) to incorporate the complete form factor has major repercussions for the sensitivity of planned searches targeting high and low frequency axions, including those utilizing novel cavity geometries [18,19].

The modified Maxwell's equations accounting for an axion field $a$ with no spatial dependence in the presence of a dc magnetic field as shown in Fig. 1 are given by [7]

$$
\begin{gathered}
\nabla \cdot \vec{E}=0, \quad \nabla \cdot \vec{B}=0, \quad \nabla \times \vec{E}=-\frac{\partial \vec{B}}{\partial t}, \\
\nabla \times \vec{B}=\frac{1}{c^{2}} \frac{\partial \vec{E}}{\partial t}-g_{\alpha \gamma \gamma} \frac{\vec{B}_{0}}{c} \frac{\partial a}{\partial t},
\end{gathered}
$$

where $g_{\alpha \gamma \gamma}$ is the strength of the axion-photon coupling (equal to $g_{\gamma} \alpha / \pi f_{a}$ ), $a=a_{0} e^{-j \omega_{a} t}$, and $\vec{B}_{0}=B_{0} \overrightarrow{\hat{z}}$ (the solenoid magnetic field). For this situation $\vec{E}=0$ and $\nabla \times \vec{B}_{0}=0$; thus, Eq. (3) becomes

$$
\nabla \times \vec{B}_{a}=\frac{1}{c^{2}} \frac{\partial \vec{E}_{a}}{\partial t},
$$




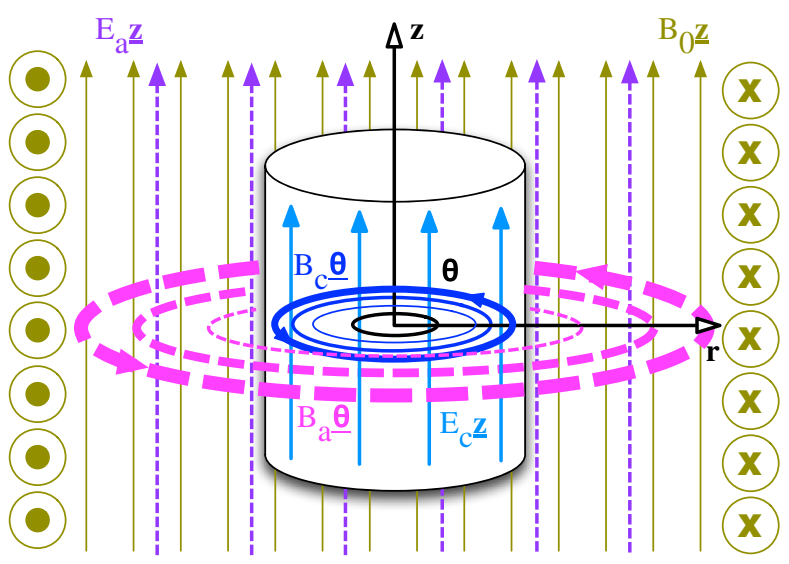

FIG. 1. Sketch of electromagnetic fields inside an axion haloscope. The solenoid (gold) produces a static magnetic field $B_{0} \overrightarrow{\hat{z}}$, which interacts with axions to produce an electric field (purple) $E_{a} \overrightarrow{\hat{z}}$ (5) and a magnetic field (magneta) $B_{a} \overrightarrow{\hat{\phi}}$ (6). For the $\mathrm{TM}_{010}$ mode the cavity supports an electric field (aqua) $E_{c} \overrightarrow{\hat{z}}$ and a magnetic field (blue) $B_{c} \overrightarrow{\hat{\phi}}$.

where $\vec{B}_{a}$ is the magnetic field component of the photons produced via the axion-photon coupling. The right-hand side of Eq. (3) defines the electric field generated by the axions as

$$
\vec{E}_{a}=-g_{\alpha \gamma \gamma} a c B_{0} \overrightarrow{\hat{z}}
$$

Applying Stokes' theorem inside the solenoid, from Eqs. (4) and (5) we conclude that

$$
\vec{B}_{a}=-\frac{g_{\alpha \gamma \gamma}}{2 c} r B_{0} \frac{\partial a}{\partial t} \overrightarrow{\hat{\phi}}
$$

where $r$ is the distance from the center of the solenoid in the radial direction.

We now consider a cylindrical cavity with resonant electric and magnetic mode field structures defined as $\vec{E}_{c}$ and $\vec{B}_{c}$, respectively. The electric energy stored in the cavity mode is given by

$$
U_{e}=\frac{1}{4} \epsilon_{0} \int d V_{c}\left|E_{c}\right|^{2},
$$

while the magnetic energy stored in the cavity mode is given by

$$
U_{m}=\frac{1}{4} \frac{1}{\mu_{0}} \int d V_{c}\left|B_{c}\right|^{2} .
$$

Now we consider the electric and magnetic energy converted from axions. From the effective axion electric field (5) the electric energy converted into the cavity is

$$
U_{a, e}=\frac{1}{4} \epsilon_{0} g_{\alpha \gamma \gamma} a c B_{0} \int d V_{c} \vec{E}_{c} \cdot \overrightarrow{\hat{z}},
$$

while from the effective axion magnetic field (6) the magnetic energy converted into the cavity is

$$
U_{a, m}=\frac{1}{4} \frac{1}{\mu_{0}} \frac{g_{\alpha \gamma \gamma}}{c} B_{0} \frac{\partial a}{\partial t} \int d V_{c} \frac{r}{2} \vec{B}_{c} \cdot \overrightarrow{\hat{\phi}} .
$$

By equating the electric energy deposited into the cavity by the axions [Eq. (9)] with the electric energy stored in the cavity mode [Eq. (7)] we can express the electric energy in the cavity due to axion conversion as

$$
U_{a, e}=\frac{1}{2} c^{2} \epsilon_{0} g_{\alpha \gamma \gamma}^{2} a_{\mathrm{rms}}^{2} B_{0}^{2} V C_{E},
$$

where we have defined the cavity mode-dependent electric form factor as

$$
C_{E}=\frac{\left|\int d V_{c} \vec{E}_{c} \cdot \overrightarrow{\hat{z}}\right|^{2}}{V \int d V_{c}\left|E_{c}\right|^{2}} .
$$

This is the standard axion haloscope form factor commonly denoted by $C$ throughout the literature [see Eq. (1)]. It has been implicitly assumed that the magnetic field contributes the same amount of energy and so a factor of 2 has been applied to Eq. (11) in the past to calculate the sensitivity of haloscope experiments. Now, in this work we explicitly consider the magnetic field contribution. Proceeding as before, by considering Eqs. (8) and (10) we can express the magnetic energy in the cavity due to axion conversion as

$$
U_{a, m}=\frac{1}{2} \frac{1}{\mu_{0}} g_{\alpha \gamma \gamma}^{2} a_{\mathrm{rms}}^{2} B_{0}^{2} V C_{B},
$$

where we define the cavity mode-dependent magnetic form factor as

$$
C_{B}=\frac{\frac{\omega_{a}^{2}}{c^{2}}\left|\int d V_{c} \frac{r}{2} \vec{B}_{c} \cdot \overrightarrow{\hat{\phi}}\right|^{2}}{V \int d V_{c}\left|B_{c}\right|^{2}} .
$$

It is worth emphasizing that $r$ and $\phi$ refer to the solenoid coordinates, not the cavity. The total electromagnetic energy stored in the cavity will be the sum of the electric and magnetic contributions given by Eqs. (11) and (13)

$U_{a, e m}=U_{a, e}+U_{a, m}=\frac{1}{2} \frac{1}{\mu_{0}} g_{\alpha \gamma \gamma}^{2} B_{0}^{2} V a_{\mathrm{rms}}^{2}\left(C_{E}+C_{B}\right)$.

To obtain the signal power expected on resonance, we multiply Eq. (15) by $\omega_{a} Q_{L}$ such that

$$
\begin{aligned}
P_{a} & =\frac{1}{\mu_{0}} g_{\alpha \gamma \gamma}^{2} a_{\mathrm{rms}}^{2} \omega_{a} V B_{0}^{2} Q_{L} C_{\mathrm{EM}} \\
& =g_{\alpha \gamma \gamma}^{2} \frac{\rho_{a}}{m_{a}} V B_{0}^{2} Q_{L} C_{\mathrm{EM}}
\end{aligned}
$$




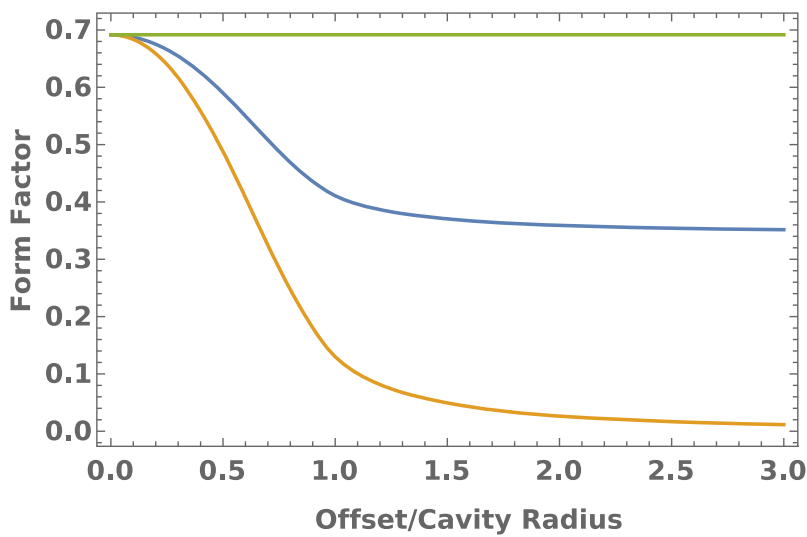

(a)

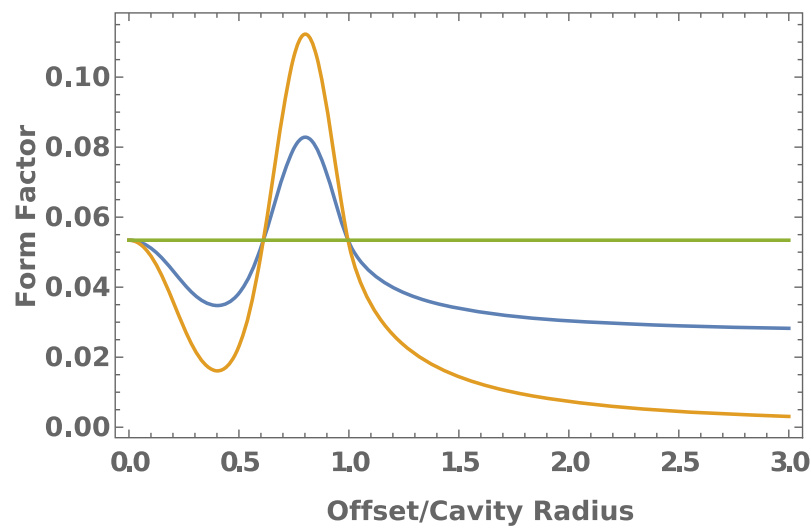

(c)

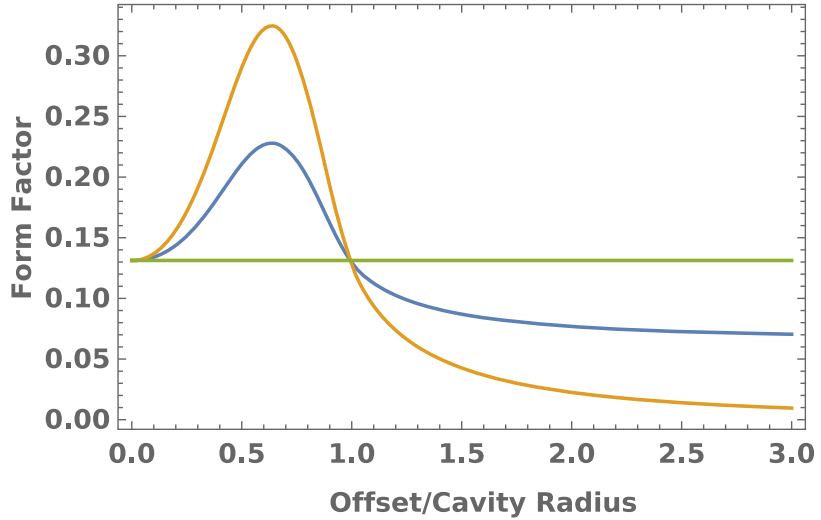

(b)

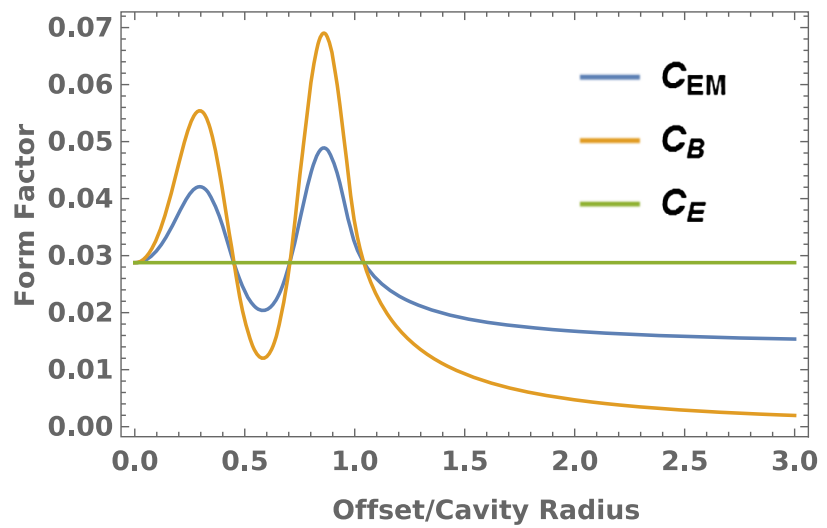

(d)

FIG. 2. Electric form factor (green), magnetic form factor (orange), and electromagnetic form factor (blue) as a function of the offset of the cavity center from the solenoid center, normalized to the cavity radius $(e / a)$. Results are presented for the following modes: (a) $\mathrm{TM}_{010}$, (b) $\mathrm{TM}_{020}$, (c) $\mathrm{TM}_{030}$, and (d) $\mathrm{TM}_{040}$.

(see Ref. [20]); here the axion frequency $\omega_{a}$ is equal to the cavity resonance frequency and $C_{\mathrm{EM}}$ is the complete electromagnetic form factor $\left(C_{E}+C_{B}\right) / 2$. For the standard haloscope design, where the cavity resonant TM mode is axially symmetric with respect to the central axis of the applied magnetic field, the electric and magnetic form factors are equal $\left(C_{E}=C_{B}\right)$ and Eq. (16) reverts to Eq. (1). However, due to the $r$ dependence in $C_{B}$ [Eq. (14)] it is clear that for more general experiments with cavities offset from axial symmetry, or with separated electric and magnetic fields, the values of $C_{E}$ and $C_{B}$ would not be equal, and the complete electromagnetic form factor would need to be calculated. For example, efforts to search for higher frequency axions can involve power-summing multiple cavities within a solenoid [15,21]; each of these nominally identical cavities will produce different amounts of power depending upon their relative location within the solenoid.

To explore the sensitivity of a single resonant cavity offset from the center of a solenoid implies that the radius of the cavity is smaller than that of the solenoid. Such a setup is most applicable to higher frequency axion searches, either with a single cavity or multiple cavities.
Considering the radial dependence of the magnetic form factor defined in Eq. (14), and understanding that this relates to the radial value of the solenoid's natural cylindrical coordinate system, we must perform a coordinate transform in order for the dot product of the axion and cavity fields to be physically meaningful. This gives the following expression for the magnetic form factor

$$
C_{B}=\frac{\frac{\omega_{a}^{2}}{c^{2}}\left|\int d V_{c} B_{c_{\phi}} \frac{r-e \cos \phi}{2}\right|^{2}}{V \int d V_{c}\left|B_{c}\right|^{2}},
$$

where $B_{c_{\phi}}$ is the $\phi$ component of the cavity magnetic field, $r$ and $\phi$ are the solenoid's natural radial and azimuthal coordinates, and $e$ is the offset of the center of the cavity from the center of the solenoid. We could perform a similar transformation for the electric field, but for the $\mathrm{TM}_{0 \times 0}$ mode family as employed by haloscope experiments it is trivial as the $z$ directions of the two systems are the same. Form factors for the $\mathrm{TM}_{0 x 0}$ modes were computed numerically as a function of $e / a$, where $a$ is the cavity radius. Figure 2 shows plots of the magnetic form factor $C_{B}$, the electric form factor $C_{E}$, and the complete electromagnetic form factor $C_{\mathrm{EM}}$ 
$\left(=\left(C_{B}+C_{E} / 2\right)\right)$ for various TM modes as a function of $e / a$. While the traditional electric form factor remains constant for all modes as expected, the electromagnetic form factor decreases with offset from the center for the $\mathrm{TM}_{010}$ mode, but for higher order modes we see positions with an increased form factor. For a $\mathrm{TM}_{010}$ mode, as the cavity moves away from the center of the solenoid, some of the $\phi$ direction magnetic field of the cavity, which was previously in phase with the axion field, is now opposing the magnetic field of the axion (see Fig. 1). The cancellation of the fields leads to a reduced mode overlap and lower sensitivity. However, for higher order modes with alternating in and out of phase magnetic field components, as the cavity is offset from the center there are regions where more of the cavity magnetic field is in the same direction as the axion magnetic field (as it was previously in the opposite direction when the cavity was central), thus achieving a higher overlap and a higher form factor. The greatest improvement can be seen in the $\mathrm{TM}_{020}$ mode, with a sensitivity increase of $\sim 75 \%$. Clearly, great care must be taken when positioning cavities within the solenoid to avoid reduced sensitivity to axions. However, this also opens up the potential to increase the sensitivity of haloscope axion searches that use higher order modes, noting that with the right positioning the product of the cavity volume and electromagnetic form factor can be larger than that of the fundamental mode.

While the magnetic coupling of the axion has been considered before in principle, the full implication of this coupling for many current and future proposed axion searches has been frequently overlooked and not treated explicitly. Any cavity-based axion detection scheme must consider this coupling, as even a slight deviation from a perfectly symmetric central placement of cavities in a magnetic field will alter the electromagnetic form factor. For example, mechanisms to tune the resonant frequency of a cavity by perturbing the electromagnetic field geometry with metallic or dielectric rods will impact the electric and magnetic couplings in different ways-it is not sufficient to only calculate the electric form factor.

Detection of axions via the magnetic coupling was previously proposed through the use of an $L C$ circuit combined with a sensitive magnetometer as the read-out mechanism [17]. However, this treatment did not generalize the problem for electromagnetic resonant detection. The electrical analogue to the magnetometer proposal would be to detect separately the electric field generated by an axion using an electrometer. In contrast, the generalized form factor technique is applicable to any electromagnetic resonant system. By defining the complete electromagnetic form factor, we have opened the possibility of calculation of the sensitivity of systems with separated electric and magnetic fields, such as lumped element $L C$ resonators, by implementing standard low noise amplifier read-outs. Such lumped systems are inherently lower in frequency than standard axion cavity resonator haloscopes, which are limited by the volume available within a high field magnet to about $500 \mathrm{MHz}$. In particular, the resonant frequencies of $3 \mathrm{D}$ lumped element $L C$ resonators, commonly known as reentrant cavities [22-25], can access the preinflationary [26] low mass axion range below the bounded Axion Dark Matter Experiment mass range [9,13]. Large areas of this region currently have no mature proposals for detection. These 3D $L C$ structures can be engineered to tune over large ranges of frequency space within a given physical cavity size; however, the spatial separation of the electric and magnetic resonant fields necessitates the use of the complete electromagnetic form factor to calculate the contribution of both the electric and magnetic components.

In conclusion, an axion in the presence of a magnetic field will convert into a real photon with electric and magnetic field components. We have shown how the generated magnetic field component interacts with a resonant cavity structure, such as those utilized in axion haloscope searches. In doing so we have defined the complete electromagnetic form factor for axion haloscopes, which has major repercussions for the sensitivity of future axion experiments and opens up new opportunities to search for low and high mass dark matter axions.

The authors thank Ian McArthur for useful discussions. This work was supported by Australian Research Council Grants No. CE110001013 and No. DP130100205, as well as the Australian Postgraduate Award and the Bruce and Betty Green Foundation.

*ben.mcallister@uwa.edu.au †michael.tobar@uwa.edu.au

[1] R. D. Peccei and H. R. Quinn, Phys. Rev. Lett. 38, 1440 (1977).

[2] L. F. Abbott and P. Sikivie, Phys. Lett. 120B, 133 (1983).

[3] J. Preskill, M. B. Wise, and F. Wilczek, Phys. Lett. 120B, 127 (1983).

[4] M. Dine and W. Fischler, Phys. Lett. 120B, 137 (1983).

[5] J. Ipser and P. Sikivie, Phys. Rev. Lett. 50, 925 (1983).

[6] L. J. Rosenberg, Proc. Natl. Acad. Sci. U.S.A. 112, 12278 (2015).

[7] P. Sikivie, Phys. Rev. Lett. 51, 1415 (1983).

[8] P. Sikivie, Phys. Rev. D 32, 2988 (1985).

[9] J. Hoskins, J. Hwang, C. Martin, P. Sikivie, N. S. Sullivan, D. B. Tanner, M. Hotz, L. J. Rosenberg, G. Rybka, A. Wagner, S. J. Asztalos, G. Carosi, C. Hagmann, D. Kinion, K. van Bibber, R. Bradley, and J. Clarke, Phys. Rev. D 84, 121302 (2011).

[10] J. E. Kim, Phys. Rev. Lett. 43, 103 (1979).

[11] M. Shifman, A. Vainshtein, and V. Zakharov, Nucl. Phys. B166, 493 (1980).

[12] M. Dine, W. Fischler, and M. Srednicki, Phys. Lett. 104B, 199 (1981).

[13] S. J. Asztalos, G. Carosi, C. Hagmann, D. Kinion, K. van Bibber, M. Hotz, L. J. Rosenberg, G. Rybka, J. Hoskins, 
J. Hwang, P. Sikivie, D. B. Tanner, R. Bradley, and J. Clarke, Phys. Rev. Lett. 104, 041301 (2010).

[14] O. K. Baker, M. Betz, F. Caspers, J. Jaeckel, A. Lindner, A. Ringwald, Y. Semertzidis, P. Sikivie, and K. Zioutas, Phys. Rev. D 85, 035018 (2012).

[15] T. M. Shokair, J. Root, K. A. Van Bibber, B. Brubaker, Y. V. Gurevich, S. B. Cahn, S. K. Lamoreaux, M. A. Anil, K. W. Lehnert, B. K. Mitchell, A. Reed, and G. Carosi, Int. J. Mod. Phys. A 29, 1443004 (2014).

[16] L. H. Nguyen, D. Horns, A. Lobanov, and A. Ringwald, arXiv:1511.03161.

[17] P. Sikivie, N. Sullivan, and D. B. Tanner, Phys. Rev. Lett. 112, 131301 (2014).

[18] R. Seviour, I. Bailey, N. Woollett, and P. Williams, J. Phys. G 41, 035005 (2014).

[19] G. Rybka, A. Wagner, K. Patel, R. Percival, K. Ramos, and A. Brill, Phys. Rev. D 91, 011701 (2015).
[20] E. J. Daw, Ph.D. thesis, Massachusetts Institute of Technology, 1998.

[21] D. S. Kinion, Ph.D. thesis, University of California at Davis, 2001.

[22] Y. Fan, Z. Zhang, N. Carvalho, J.-M. Le Floch, Q. Shan, and M. Tobar, IEEE Trans. Microwave Theory Tech. 62, 1657 (2014).

[23] J.-M. Le Floch, Y. Fan, M. Aubourg, D. Cros, N. C. Carvalho, Q. Shan, J. Bourhill, E. N. Ivanov, G. Humbert, V. Madrangeas, and M.E. Tobar, Rev. Sci. Instrum. 84, 125114 (2013).

[24] N. C. Carvalho, Y. Fan, J.-M. Le Floch, and M. E. Tobar, Rev. Sci. Instrum. 85, 104705 (2014).

[25] M. Goryachev and M. E. Tobar, New J. Phys. 17, 023003 (2015).

[26] E. Berkowitz, M. I. Buchoff, and E. Rinaldi, Phys. Rev. D 92, 034507 (2015). 